\title{
ON BARELY CONTINUOUS FUNCTIONS
}

\author{
RICHARD STEPHENS \\ Department of Applied Mathematics \\ Western Carolina University \\ Cullowhee, NC 28723 \\ (Received March 23, 1987)
}

ABSTRACT. The term barely continuous is a topological generalization of Baire-1 according to F. Gerlits of the Mathematical Institute of the Hungarian Academy of Sciences, and thus worthy of further study. This paper compares barely continuous functions and continuous functions on an elementary level. Knowing how the continuity of the identity function between topologies on a given set yields the lattice structure for those topologies, the barely continuity of the identity function between topologies on a given set is investigated and used to add to the structure of that lattice. Included are certain sublattices generated by the barely continuity of the identity function between those topologies. Much attention is given to topologies on finite sets.

KEY WORDS AND PHRASES. Barely continuous, barely finer, slightly finer, barely equivalent, up lattice, down lattice, barely discrete. 1980 AMS SUBJECT CLASSIFICATION CODE. 54C10, 54H12.

\section{INTRODUCTION.}

The principle results are: (1) Necessary conditions for the equivalence of continuous and barely continuous. (2) Slightly finer as an indicator of "nearness" of one topology to another in the lattice of topologies. (3) No non-trivial regular topology on a finite set has an up lattice or a down lattice. Examples are included to give the reader a better picture of barely continuous.

2. DEFINITION AND EXAMPLES.

A function $f$ from a topological space $X$ to a topological space $Y$ is barely continuous if for each closed subset $A$ of $X$, the restriction of $f$ to $A$ with its relative topology has at least one point of continuity (Kuratowski [1]).

Let each of the following topologies be defined on the closed interval $[0,1]$, and let each arrow denote the identity function. Let $u$ be the usual topology, cc be the countable complement topology, cf be the co-finite topology, [0,x) be the topology with basic open sets of this form, and nc be the naive one point compactification of $[0,1)$ with the usual topology. The following statements are easily verified if we take b.c. to mean barely continuous. 
(1)

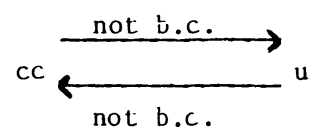

(2)

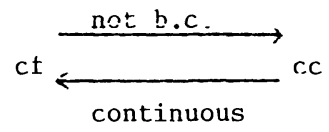

(3)

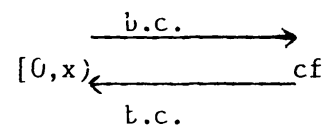

(4)

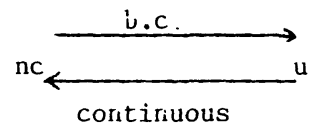

(5)

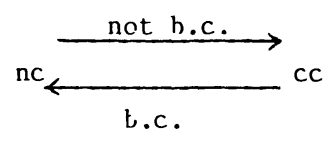

$(6)$

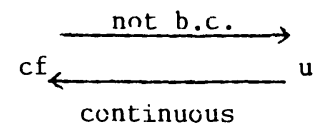

(7)

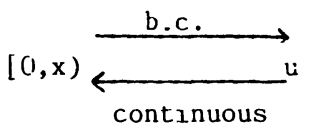

(8)

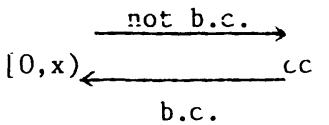

(9)

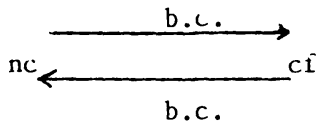

(10)

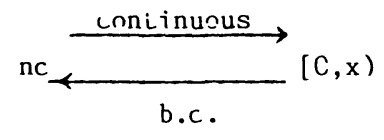

TABLE 1

3. NECESSARY CONDITIONS FOR BARELY CONTINUITY TO IMILY CONTINUITY.

THEOREM 2. If each barely continuous function from a tcpoiogical space $X$ into a topological space $\mathrm{Y}$ is continuous, then $\mathrm{Y}$ is indiscrete or every closed set in $\mathrm{X}$ is also open.

PROOF. We will show that the contrapositive of this theorem is true. Suppose $\mathrm{Y}$ is not indiscrete, and let $\mathrm{V}$ be an open set in $\mathrm{Y}$ such that $\mathrm{V} \neq \mathrm{Y}$. Since every closed set in $X$ is open if and only if every open set in $X$ is closed, let $U$ be an open set in $X$ such that $U$ is not closed. Let $\mathrm{v}$ be an element of $\mathrm{V}$ and $\mathrm{w}$ be an element of the complement of $V$. Define a function $f$ from $X$ into $Y$ such that $f$ maps $U$ to $w$ and the complement of $U$ to $v$. If $A$ is a closed subset of $X$, then $A$ intersects $U$ or $A$ is a subset of the complement of $U$. In each case, $\left.f\right|_{A}$ is a constant on an open subset of $\mathrm{A}$, and so $\mathrm{f}$ is barely continuous. Now, let $\mathrm{x}$ be a limit point of $U$ such that $x$ is not an element of $U$, and let $U_{x}$ be an open set containing $x$. Then, $v$ and $w$ are elements of $f\left(U_{x}\right)$, with $f(x)=v \varepsilon v$, and $f\left(U_{x}\right)$ not a subset of $V$. Hence, $f$ is not continuous.

One may easily construct counterexamples to show that the conditions of THEOREM 2 are not sufficient.

DEFINITION 3. A topological space $(\mathrm{X}, \mathrm{T})$ is barely discrete if each function from $(X, T)$ to any topological space is barely continuous.

It is easily seen that a topological space $(X, T)$ is barely discrete if and on1y if the identity function from $(X, T)$ to ( $X$, discrete) is barely continuous.

4. LATTICE PROPERTIES.

Let $X$ and $Y$ be topological spaces on the same set.

DEFINITION 4. $X$ is barely finer than $Y$ ( $Y$ is barely coarser than $X$ ) if the identity map from $X$ to $Y$ is barely continuous. We write $X[>] Y$, or $Y[<] X$.

DEFINITION 5. $X$ is slight1y finer than $Y$ ( $Y$ is slightly coarser than $\dot{X}$ ) if $X$ is finer than $Y$ and $Y$ is barely finer than $X$. We write $X[\geq] Y$, or $Y[s] X$. 
DEFINITION 6. $\mathrm{X}$ is barely equivalent to $\mathrm{Y}$ if $\mathrm{X}$ is barely finer than $\mathrm{Y}$ and $\mathrm{Y}$ is barely finer than $X$. We write $X[=] Y$, or $Y[=] X$.

DEFINITION 7. A topology $T$ on $X$ is an ultraspace if the only topology on $X$ strictly finer than $T$ is the discrete topology. The ultraspaces on a set $X$ are of the following form. Let $x$ be an element of $X$, and $U$ be an ultrafilter on $X$, then a subset $V$ of $X$ is open if $x$ is not an element of $V$ or if $V$ is a member of $U$ which contains $x$ (Fröhlich [2]).

THEOREM 8. Each ultraspace on a set $X$ is barely discrete.

PROOF. Let $(x, U)$ be an ultraspace on $X, Y$ be a topological space, $f$ be a function from $X$ to $Y$, and $A$ be a closed subset of $X$. Then $X=A$, or $A-\{x\}$ has the discrete topology. In either case, it is clear that $\left.f\right|_{A}$ has a point of continuity. Hence, $f$ is barely continuous.

Suppose $X$ has at least two elements. Then the identity map from ( $X$, indiscrete) to ( $X$, discrete) is not barely continuous. A1so, the only topology on $X$ coarser than all the ultraspaces is the indiscrete topology. Since the identity is barely continuous on each ultraspace, there is no coarsest topology on $\mathrm{X}$ for which the discrete topology is slightly finer. From this, it is clear that there is no coarsest topology on $X$ which is barely discrete. Also, if $f$ is a function which is barely continuous from $\left(X, T_{a}\right)$ to a topological space $Y$ for each a in some index set $A$, then $f$ is not necessarily barely continuous from (X, $\inf \left\{T_{a}\right\}$ ) to $Y$.

LEMMA 9. Let $X, Y$, and $Z$ be topological spaces. If $f$ is a barely continuous function from $X$ to $Y$, and $g$ is a continuous function from $Y$ to $Z$, then gof $=g(f)$ is a barely continuous function from $X$ to $Z$.

PROOF. Under the assumptions of the lemma, let $A$ be a closed subset of $X$, and a be an element of $A$ such that $\left.f\right|_{A}$ is continuous at a. Hence, gof is barely continuous, since $\left.(g \circ f)\right|_{A}=g\left(\left.f\right|_{A}\right)$ is continuous at a.

We now give an example to show that under the conditions of the lemma, if $f$ is continuous and $\mathrm{g}$ is barely continuous, then gof is not necessarily barely continuous. Let $\mathrm{f}$ and $\mathrm{g}$ be identity functions, let $\mathrm{X}=\mathrm{Y}=\mathrm{Z}=\{\mathrm{a}, \mathrm{b}, \mathrm{c}\}$, the topology on $X$ be $\{\phi,\{c\},\{a, b\}, X\}$, the topology on $Y$ be the indiscrete topology, and the topology on $Z$ be $\{\phi,\{a\},\{b\},\{a, b\}, Z\}$. Now, $f$ is continuous from $X$ to $Y$, and $\mathrm{g}$ is barely continuous from $\mathrm{Y}$ to $\mathrm{Z}$, but gof is not barely continuous from $\mathrm{X}$ to $\mathrm{Z}$ since $\left.(g \circ f)\right|_{\{a, b\}}$ is neither continuous at a nor at $b$.

This property of slightly finer can be used as an indicator of how far above one topology another topology happens to be in the lattice of topologies on a given set. It follows from Lemma 9 , if $T$ and $S$ are topologies on a set $X$, and $T$ is slightly finer than $S$, then each topology on $X$ between $T$ and $S$ is slightly finer than $S$. Also, if $T$ is finer than $S$, and $T$ is not slightly finer than $S$, then each topology finer than $T$ is not slightly finer than $S$. Thus, within a chain of topologies on $X$, if we consider all topologies which are finer than $S$, the topologies which are slightly finer than $S$ are closer to $S$ than the topologies which are not slightly finer than S. This is more formally stated in the next theorem.

THEOREM 10. Suppose $S, T$, and $W$ are topologies on a set $X$ such that $W$ is finer than $T$, and $T$ is finer than $S$. If $W$ is slightly finer than $S$, then $T$ is slightly 
finer than S. (Also, if $T$ is not slightly finer than $S$, then $W$ is not slightly finer than S.)

PROOF. Let $S, T$, and $W$ be topologies on a set $X$ so that $W$ is finer than $T$, and $T$ is finer than $S$. Suppose $W$ is slightly finer than $S$, then the identity map from $(X, S)$ to $(X, W)$ is barely continuous. Since the identity map from $(X, W)$ to $(X, T)$ is continuous, it follows from Lemma 9 that the identity map from $(X, S)$ to $(X, T)$ is barely continuous. Hence, $\mathrm{T}$ is slightly finer than $\mathrm{S}$.

COROLLARY 11. Let $S, T$, and $W$ be members of the same chain in the lattice of topologies on a set $X$. If $W$ is finer than $S$, but not slightly finer than $S$, and $T$ is slightly finer than $S$, then $W$ is finer than $T$.

Upon an investigation of the lattices of topologies on a three element set, we find that barely equivalence is not an equivalence relation. However, any two $t_{1}$ topologies on a set $X$ are linked by a "chain" of topologies so that each link in the "chain" consists of two barely equivalent $t_{1}$ topologies.

DEFINITION 12. Two topologies, $T$ and $W$, on a set $X$ are p-weakly equivalent if there exist a finite set of topologies, $\mathrm{T}_{1}, \mathrm{~T}_{2}, \ldots, \mathrm{T}_{\mathrm{n}}$, on $\mathrm{X}$ so that $\mathrm{T}$, $\mathrm{W}, \mathrm{T}_{1}, \mathrm{~T}_{2}, \ldots, \mathrm{T}_{\mathrm{n}}$, each have property $\mathrm{p}$, and $\mathrm{T}[=] \mathrm{T}_{1}[=] \mathrm{T}_{2}[=] \ldots[=] \mathrm{T}_{\mathrm{n}}[=] \mathrm{W}$.

THEOREM 13. If $T$ and $W$ are $t_{1}$ topologies on a set $X$, then $T$ and $W$ are $t_{1}$-weak1y equivalent.

PROOF. Consider the $t_{1}$ topologies on a set $X$. This set of topologies forms a complete sublattice of topologies on $\mathrm{X}$. The finest $\mathrm{t}_{1}$ topology is the discrete topology, and the coarsest $t_{1}$ topology is the co-finite topology. Let $T_{c}$ be the co-finite topology, $T$ be a $t_{1}$ topology, $x$ be an element of $X$, and $S$ be the topology consisting of each member of $\mathrm{T}$ to which $\mathrm{x}$ does not belong and each member of $T_{C}$ to which $x$ does belong. We see that $S$ is a $t_{1}$ topology, $T$ is slight $1 y$ finer than $S$, and $S$ is slightly finer than $T_{c}$. Thus, $T_{c}$ is $t_{1}$-weakly equivalent to $\mathrm{T}$ since slightly finer implies barely equivalent. Hence, any $t_{1}$ topology is $t_{1}$-weakly equivalent to $T_{c}$, and thus any two $t_{1}$ topologies are $t_{1}$-weakly equivalent to each other.

5. SUBLATTICES.

Consider again the lattice of topologies on a set X. For each topology $T$ on $X$, let $\mathrm{T} \downarrow$ denote the collection of topologies which are slightly coarser than $T$, and let $\mathrm{T} \uparrow$ denote the collection of topologies which are slightly finer than $\mathrm{T}$. Read $\mathrm{T} \downarrow$ as $\mathrm{T}$-down, and $\mathrm{T} \uparrow$ as $\mathrm{T}$-up.

DEFINITION 14. Let $T$ be a topology on a given set $X$. If $T \downarrow$ forms a sublattice of the lattice of topologies on $X$, then call that sublattice the down lattice for $T$. If $\mathrm{T} \uparrow$ forms a sublattice, then call that sublattice the up lattice for $\mathrm{T}$.

We find that for a three element set each down lattice has the indiscrete topology for its coarsest member and each up lattice has the discrete topology for its finest member. Also, each topology on a three element set which is not a non-trivial regular topology has either a down lattice or an up lattice, and each non-regular topology (on a three element set) which has the same number of open sets as each non-trivial regular topology (on a three element set) has both a down lattice and an up lattice. Previous results show that for a set with more than one element, 
the discrete topology has no down lattice, and the following lemma shows that for a finite set with more than one element, the indiscrete topology has no up lattice.

LEMMA 15. For a finite set, the discrete topology is the finest member of any up lattice.

PROOF. Let $T$ be a topology on a finite set $X$. Let $x_{1}, \ldots, x_{n}$ be the members of $(X, T)$ which are not open sets as singleton sets. For each $i=1,2, \ldots n$, let $T_{i}$ be the topology whose base is formed by taking the base for $T$ and including $x_{i}$. We find that $T_{i}$ is slightly finer than $T$ for each $i=1,2, \ldots, n$. Also, the only topology that is finer than $T_{i}$ for each $i=1,2, \ldots, n$ is the discrete topology. Thus, if $\mathrm{T}$ has an up lattice, then the finest member of that up lattice is the discrete topology.

LEMMA 16. For a finite set, the indiscrete topology is the coarsest member of any down lattice.

PROOF. Let $T$ be a topology on a finite set $X$, with subbasis $B=$ $\left\{v_{1}, \ldots, v_{m}\right\}$. For each $i=1,2, \ldots, m$, let $T_{i}$ be the topology with $B-$ $\left\{V_{i}\right\}$ as its subbasis. Suppose $A$ is a closed subset of $\left(X, T_{i}\right)$.

Case (i) A is a subset of $V_{i}$. In this case the topology on $A$ induced by $T_{i}$ is the same as the topology on $A$ induced by $T$.

Case (ii) $A$ is not a subset of $V_{i}$. In this case the topology on $A-\left\{V_{i}\right\}$ induced by $T_{i}$ is the same as the topology on $A-\left\{V_{i}\right\}$ induced by $\mathrm{T}$.

In either case, the identity map from $\left(X, T_{i}\right)$ to $(X, T)$ is seen to be barely continuous, and the only topology coarser than $\mathrm{T}_{i}$ for each $i=1,2, \ldots, \mathrm{m}$ is the indiscrete topology. Thus, if $\mathrm{T}$ has a down lattice, then the coarsest member of that down lattice is the indiscrete topology.

THEOREM 17. For a finite set, no non-trivial regular topology has an up lattice or a down lattice.

PROOF. In a non-trivial regular topology on a finite set $X$, each point has an open neighborhood smaller than $X$. Thus, the identity map from the indiscrete topology to such a non-trivial regular topology is not barely continuous. Also, in such a topology there is some point which has a smallest open neighborhood larger than the point itself. Since that open neighborhood is also closed, the identity map from such a topology to the discrete topology is not barely continuous. Hence, by the two preceeding lemmas, the theorem is proven.

\section{REFERENCES}

1. Kuratowski, K. Topology, Vol. 1, Academic Press, New York, 1966.

2. Frohlich, 0, Das Halbordnungssytem der topologischen Raume auf einer Menge, Math. Ann. 156 (1964), 79-95.

3. Steiner, A.K. The Lattice of Topologies: Structure and Complementation, from the author's doctoral dissertation at the University of New Mexico in 1965.

4. Michae1, E. and Namioka, I. Barely Continuous Functions, Bulletin De L'Academic Polanaise Des Sciences, Vol. XXIV, No. $10 \overline{(1976), 889-892 . ~}$ 


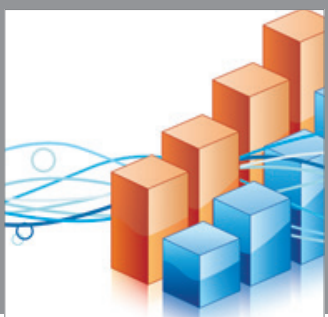

Advances in

Operations Research

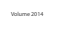

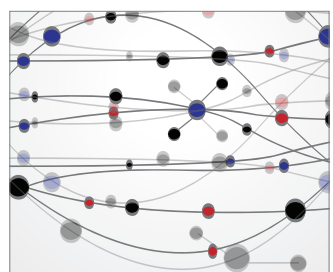

\section{The Scientific} World Journal
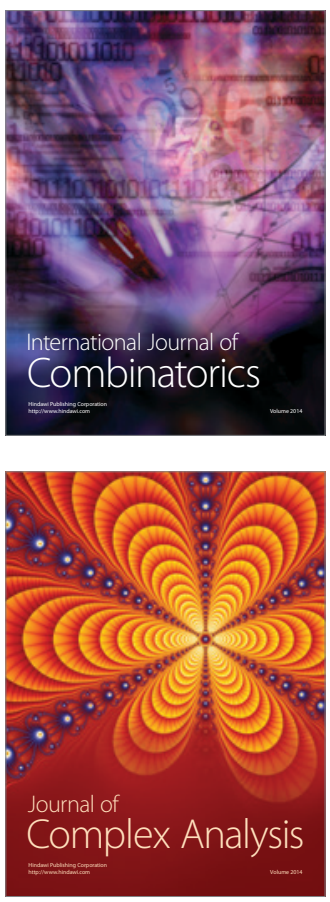

International Journal of

Mathematics and

Mathematical

Sciences
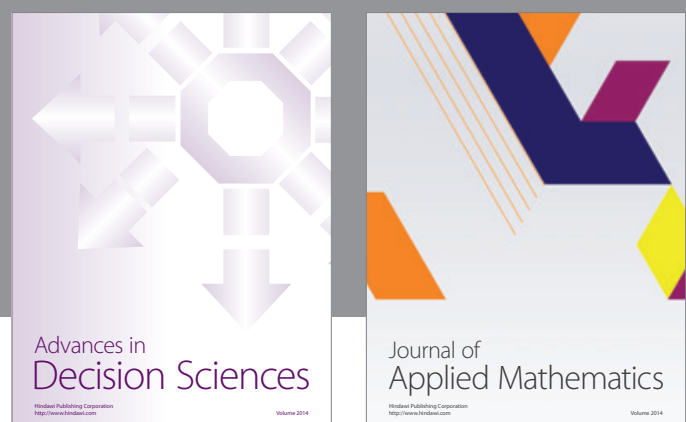

Journal of

Applied Mathematics
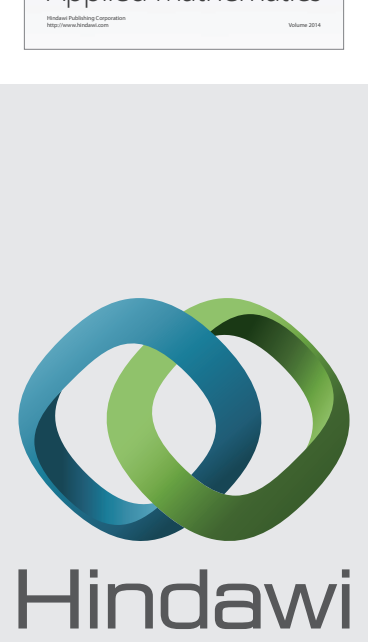

Submit your manuscripts at http://www.hindawi.com
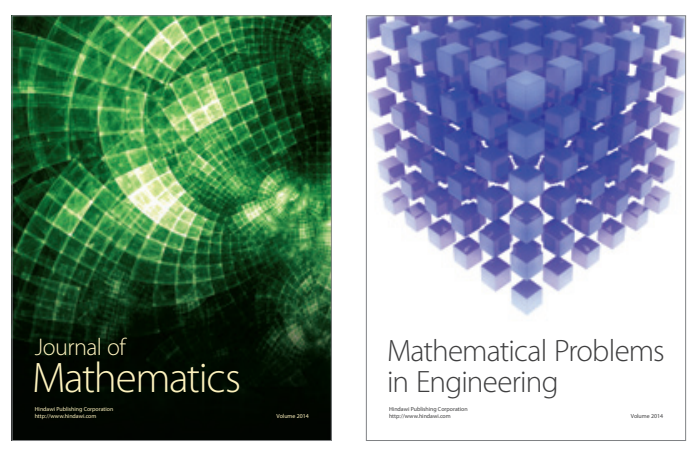

Mathematical Problems in Engineering
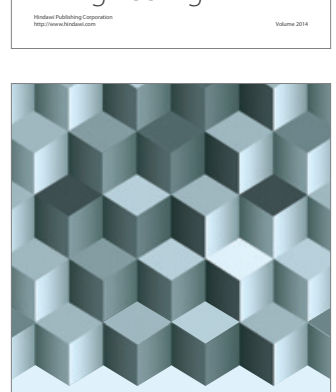

Journal of

Function Spaces
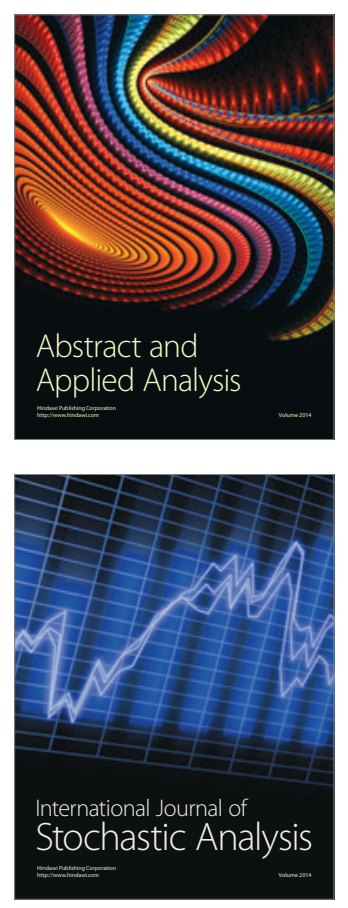

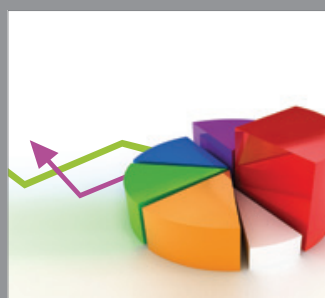

ournal of

Probability and Statistics

Promensencen
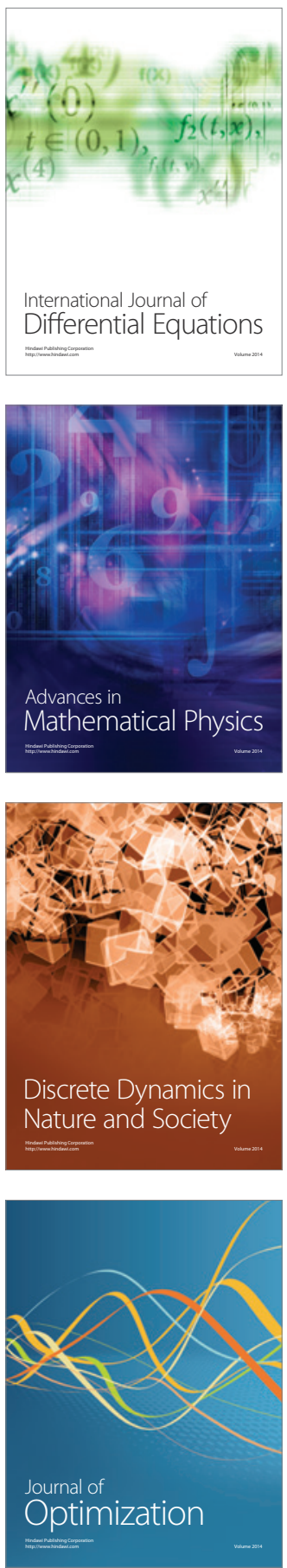